\title{
Photoconductivity in AC-driven modulated two dimensional electron gas in a perpendicular magnetic field
}

\author{
Manuel Torres \\ Instituto de Física, Universidad Nacional Autónoma de México, Apartado Postal \\ 20-364, México Distrito Federal 01000, México \\ E-mail: torres@fisica.unam.mx
}

\begin{abstract}
Alejandro Kunold
Departamento de Ciencias Básicas, Universidad Autónoma Metropolitana

Azcapotzalco, Av. San Pablo 180, México Distrito Federal 02200, México

E-mail: akb@correo.azc.uam.mx
\end{abstract}

\begin{abstract}
.
In this work we study the microwave photoconductivity of a two-dimensional electron system (2DES) in the presence of a magnetic field and a two-dimensional modulation (2D). The model includes the microwave and Landau contributions in a non-perturbative exact way, the periodic potential is treated perturbatively. The Landau-Floquet states provide a convenient base with respect to which the lattice potential becomes time-dependent, inducing transitions between the Landau-Floquet levels. Based on this formalism, we provide a Kubo-like formula that takes into account the oscillatory Floquet structure of the problem. The total longitudinal conductivity and resistivity exhibit strong oscillations, determined by $\epsilon=\omega / \omega_{c}$ with $\omega$ the radiation frequency and $\omega_{c}$ the cyclotron frequency. The oscillations follow a pattern with minima centered at $\omega / \omega_{c}=j+\frac{1}{2}(l-1)+\delta$, and maxima centered at $\omega / \omega_{c}=j+\frac{1}{2}(l-1)-\delta$, where $j=1,2,3 \ldots \ldots ., \delta \sim 1 / 5$ is a constant shift and $l$ is the dominant multipole contribution. Negative resistance states (NRS) develop as the electron mobility and the intensity of the microwave power are increased. These NRS appear in a narrow window region of values of the lattice parameter $(a)$, around $a \sim l_{B}$, where $l_{B}$ is the magnetic length. It is proposed that these phenomena may be observed in artificially fabricated arrays of periodic scatterers at the interface of ultraclean $G a A s / A l_{x} G a_{1-x} A s$ heterostructures.
\end{abstract}

PACS numbers: 72.40.+w, 73.21.Cd, 75.47.-m, 73.43.-f,

\section{Introduction.}

The microwave irradiation of two-dimensional electron systems (2DES) has remarkable consequences on the transport properties at low magnetic fields. Recently, two experimental groups 1, 2, 3, 4, reported the unexpected discovery of zero resistance 
states (ZRS) when high mobility $G a A s / A l_{x} G a_{1-x} A s$ heterostructures in weak magnetic fields were exposed to millimeter irradiation. Unlike the strong magnetic field regime, the Hall resistance is not quantized. The magnetoresistance exhibits giant oscillations, periodic in $\epsilon=\omega / \omega_{c}$ with $\omega$ the radiation frequency and $\omega_{c}$ the cyclotron frequency; the series of minima formed at $\epsilon=j+\delta, j=1,2,3 \ldots, \delta=\frac{1}{2}$ [1], 2], or $\delta=\frac{1}{4}$ [3, 4]. These discoveries triggered a large number of experimental [5, 6, 7, 8, 9, and theoretical [10, 11, 12, 13, 14, 15, 16, 17, 18, 19, 20, 21] studies. According to reference 13], ZRS probably originate from negative resistance states (NRS); it was argued that negative resistance induces the formation of current domains, yielding an instability that drives the system into a ZRS. The existence of NRS was first predicted in the pioneering work of Ryzhii [10, 11]. Nowadays two distinct mechanisms that produce negative longitudinal conductance are known: (i) the impurity scattering mechanism, which is caused by the disorder assisted absorption and emission of microwaves 10, 11, 12, 13, 14, 15, 16, 17, 18, and (ii) the distribution function mechanism, according to which the microwave absorption modifies the electron distribution function leading to a negative longitudinal conductance [5, 19, 20, 21]. A model for the impurity scattering mechanism was proposed previously by the authors [17, 18, the model is based on the fact that the microwave and Landau dynamics can be exactly taken into account producing well defined Floquet-Landau states. The disorder effects are treated perturbatively, inducing transitions between the Floquet-Landau levels. The model reproduce various of the experimentally observed features, in particular the fact that negative resistance states $(Z R S)$ appear only when the electron mobility exceeds a threshold.

Although the experiments described above do not include the effect of periodical potential modulations, exploring its physical consequences is worthwhile. We can identified at least three reasons for doing so: (i) The study of both weak [22] and strong [23] periodically modulated 2DES in the presence of magnetic fields has lead to the discovery of interesting transport effects, such as commensurability phenomena and transport anisotropies. (ii) The theoretical method previously developed in references [17, 18] is well suited to study this kind of system. (iii) The use of artificially fabricated arrays of periodic scatterers at the interface of ultraclean $G a A s / A l_{x} G a_{1-x} A s$ heterostructures [22, 23, 24, 25] may allow to test the predictions made by these theoretical studies.

In this work we make a theoretical study of the microwave photoconductivity of a 2DES in the presence of a magnetic field and a two-dimensional modulation. Two theoretical studies of modulated 2DES under the combined effects of magnetic and microwave radiation have recently appeared. Dietel et.al. [26] considered the photoconductivity in the case of 1D periodic modulation. The calculation uses first order perturbation theory for both the microwave field as well as for the periodic potential. Additionally, the calculation is restricted by the following conditions: the lattice parameter $a$ is small as compared to the cyclotron radius $R_{c}$, the temperature $T$ is large with respect to the periodic strength potential $V_{0}$, and $V_{0} \ll \hbar \omega_{c}$. Due of the 
unidirectional structure of the modulation the photocurrents parallel and perpendicular to the modulation are different. The work of Gumbs [27] applies for a strong 2D modulation, but is linear with respect to the microwave field intensity. His approach made use of the usual Kubo formula in which the matrix elements are evaluated using the numerically obtained Hofstadter-type wave functions. In this paper we address the case of a 2D modulation under different conditions; in particular we explore the possible appearance of negative resistance states. The Landau and microwave field contributions are exactly taken into account. We analyze the regime in which the following conditions hold: $k T \sim V_{0} \leq \hbar \omega_{c}$, and $\omega \tau_{t r} \sim \omega_{c} \tau_{t r}>>1$. Instead of appealing to the usual Kubo formula, our approach shows that the use of the Floquet-Landau states determines not only the wave functions required to evaluate the matrix elements; but also leads to a modified Kubo-like formula, in which the oscillations on the density of states induce the development of negative resistance states. As a first step we find a unitary transformation that exactly takes into account the dynamics associated with the Landau and radiation contributions. As a second step, the periodic potential is added perturbatively. With respect to the Landau-Floquet states, the periodic potential act as a coherent oscillating field which induces transitions between these levels. Based on this formalism, we provide a Kubo-like expression for the conductance that incorporates the oscillatory Floquet structure of the system. It is found that both $\sigma_{x x}$ and $\rho_{x x}$ exhibit strong oscillations determined by $\epsilon=\omega / \omega_{c}$. NRS develop for sufficiently high electron mobility and strong microwave power. The model is used to test chirality effects induced by the magnetic field, calculations are carried out for various E-field polarization's. Finally, we explore the nonlinear regime in which multiple photon exchange play an essential role, as well as the current-voltage characteristics of the system.

The paper is organized as follows. In the next section we present the model and the method that allow us to obtain the exact solution of the Landau-microwave system, as well as the perturbative corrections induced by the periodic potential. In section 3 we develop the formulation of the linear response theory valid in arbitrary magnetic and microwave fields. A discussion of relevant numerical calculations is presented in section (4). The last section contains a summary of our main results.

\section{The Model.}

Let us consider the motion of an electron in two dimensions subject to a uniform magnetic field $\mathbf{B}$ perpendicular to the plane and a constant electric field $\mathbf{E}_{c}$, a periodic potential $V$ and driven by microwave radiation. On the plane the dynamics is governed by the Schrödinger equation

$$
i \hbar \frac{\partial \Psi}{\partial t}=H \Psi=\left[H_{\{B, \omega\}}+V(\mathbf{r})\right] \Psi
$$

Here $H_{\{B, \omega\}}$ is written in term of the covariant derivative

$$
H_{\{B, \omega\}}=\frac{1}{2 m^{*}} \boldsymbol{\Pi}^{2}, \quad \quad \boldsymbol{\Pi}=\mathbf{p}+e \mathbf{A},
$$


where $m^{*}$ is the effective electron mass over the plane that takes into account the effects of the crystalline atomic structure over the charge carriers. The vector potential A includes all the contributions of the magnetic, electric and radiation fields:

$$
\mathbf{A}=-\frac{1}{2} \mathbf{r} \times \mathbf{B}+\operatorname{Re}\left[\frac{\epsilon E_{\omega}}{\omega} \exp \{-i \omega t\}\right]+\mathbf{E}^{c} t
$$

The superlattice potential $V(\mathbf{r})$ is decomposed in a Fourier expansion

$$
V(\mathbf{r})=\sum_{m n} V_{m n} \exp \left\{i 2 \pi\left(\frac{m x}{a}+\frac{n y}{b}\right)\right\} .
$$

We first consider the exact solution of the microwave driven Landau problem, the periodic potential effects are lately added perturbatively. Along this work we shall assume: (i) a weak modulation $|V| \ll \hbar \omega_{c}$ and (ii) the clean limit $\omega \tau_{t r} \sim \omega_{c} \tau_{t r}>>1$; here $\tau_{t r}$ is the transport relaxation time that is estimated using its relation to the electron mobility $\mu=e \tau_{t r} / m^{*}$. Based on these conditions the use of first order perturbation theory in $V$ becomes reasonable. Furthermore the second conditions justifies the use of a Fermi distribution function evaluated at the Floquet-Landau quasi-energies, see appendix (5.2).

The system posed by $H_{\{B, \omega\}}$ can be recast as a forced harmonic oscillator, a problem that was solved long time ago by Husimi 28]. Following the formalism developed in references [29, 30], we introduce a canonical transformation to new variables $Q_{\mu}, P_{\mu}$; $\mu=0,1,2$, according to

$$
\begin{aligned}
Q_{0} & =t, & P_{0} & =i \partial_{t}+e \phi+e \mathbf{r} \cdot \mathbf{E}, \\
\sqrt{e B} Q_{1} & =\Pi_{y}, & \sqrt{e B} P_{1} & =\Pi_{x}, \\
\sqrt{e B} Q_{2} & =\Pi_{x}+e B y, & \sqrt{e B} P_{2} & =\Pi_{y}-e B x .
\end{aligned}
$$

It is easily verified that the transformation is indeed canonical, the new variables obey the commutation rules: $-\left[Q_{0}, P_{0}\right]=\left[Q_{1}, P_{1}\right]=\left[Q_{2}, P_{2}\right]=i B$; all other commutators being zero. The inverse transformation gives $x=l_{B}\left(Q_{1}-P_{2}\right)$, and $y=l_{B}\left(Q_{2}-P_{1}\right)$, where $l_{B}=\sqrt{\frac{\hbar}{e B}}$ is the magnetic length. The operators $\left(Q_{2}, P_{2}\right)$ can be identified with the generators of the electric-magnetic translation symmetries [31, 32. Final results are independent of the selected gauge. From the operators in Eq. (5) we construct two pairs of harmonic oscillator-like ladder operators: $\left(a_{1}, a_{1}^{\dagger}\right)$, and $\left(a_{2}, a_{2}^{\dagger}\right)$ with:

$$
a_{1}=\frac{1}{\sqrt{2}}\left(P_{1}-i Q_{1}\right), \quad a_{2}=\frac{1}{\sqrt{2}}\left(P_{2}-i Q_{2}\right),
$$

obeying: $\left[a_{1}, a_{1}^{\dagger}\right]=\left[a_{2}, a_{2}^{\dagger}\right]=1$, and $\left[a_{1}, a_{2}\right]=\left[a_{1}, a_{2}^{\dagger}\right]=0$.

It is now possible to find a unitary transformation that exactly diagonalizes $H_{\{B, \omega\}}$, it yields

$$
W^{\dagger} H_{\{B, \omega\}} W=\omega_{c}\left(\frac{1}{2}+a_{1}^{\dagger} a_{1}\right) \equiv H_{0},
$$

with the cyclotron frequency $\omega_{c}=e B / m^{*}$ and the $W(t)$ operator given by

$$
W(t)=\exp \left\{i \eta_{1} Q_{1}\right\} \exp \left\{i \xi_{1} P_{1}\right\} \exp \left\{i \eta_{2} Q_{2}\right\} \exp \left\{i \xi_{2} P_{2}\right\} \exp \left\{i \int^{t} \mathcal{L} d t^{\prime}\right\},
$$


Photoconductivity in AC-driven modulated two dimensional electron gas in a perpendicular magnetic field 5

where the functions $\eta_{i}(t)$ and $\xi_{i}(t)$ represent the solutions to the classical equations of motion that follow from the variation of the Lagrangian

$$
\mathcal{L}=\frac{\omega_{c}}{2}\left(\eta_{1}^{2}+\zeta_{1}^{2}\right)+\dot{\zeta}_{1} \eta_{1}+\dot{\zeta}_{2} \eta_{2}+e l_{B}\left[E_{x}\left(\zeta_{1}+\eta_{2}\right)+E_{y}\left(\eta_{1}+\zeta_{2}\right)\right] .
$$

It is straightforward to obtain the solutions to the equation of motion, using the expression for the electric field $\mathbf{E}=-\partial \mathbf{A} / \partial t$ with $\mathbf{A}$ given in (3). Adding a damping term that takes into account the radiative decay of the quasiparticle, they read

$$
\begin{array}{ll}
\eta_{1}=e l_{B} E_{\omega} \operatorname{Re}\left[\frac{-i \omega \epsilon_{x}+\omega_{c} \epsilon_{y}}{\omega^{2}-\omega_{c}^{2}+i \omega \Gamma_{\text {rad }}} e^{i \omega t}\right], & \eta_{2}=e l_{B} E_{\omega} \operatorname{Re}\left[\frac{\epsilon_{y} e^{i \omega t}}{i \omega}\right]+e l_{B} E_{y}^{c} t, \\
\zeta_{1}=e l_{B} E_{\omega} \operatorname{Re}\left[\frac{\omega_{c} \epsilon_{x}+i \omega \epsilon_{y}}{\omega^{2}-\omega_{c}^{2}+i \omega \Gamma_{\text {rad }}} e^{i \omega t}\right], & \zeta_{2}=-e l_{B} E_{\omega} R e\left[\frac{\epsilon_{x} e^{i \omega t}}{i \omega}\right]-e l_{B} E_{x}^{c} t .
\end{array}
$$

According to the Floquet theorem, the wave function can be written as $\Psi(t)=$ $\exp \left(-i \mathcal{E}_{\mu} t\right) \phi_{\mu}(t)$, where $\phi_{\mu}(t)$ is periodic in time, i.e. $\phi_{\mu}\left(t+\tau_{\omega}\right)=\phi_{\mu}(t)$, with $\tau_{\omega}=2 \pi / \omega$. From Eq. (8) it is noticed that the transformed wave function $\Psi^{W}=W \Psi$ contains the phase factor $\exp \left(i \int^{t} \mathcal{L} d t^{\prime}\right)$. It then follows that the quasienergies and the Floquet modes can be deduced if we add and subtract to this exponential a term of the form $\frac{t}{\tau_{\omega}} \int_{0}^{\tau_{\omega}} \mathcal{L} d t^{\prime}$. Hence, the quasienergies can be readily read off

$$
\mathcal{E}_{\mu}=\mathcal{E}_{\mu}^{(0)}+\mathcal{E}_{\text {rad }} ; \quad \mathcal{E}_{\mu}^{(0)}=\hbar \omega_{c}\left(\frac{1}{2}+\mu\right), \quad \mathcal{E}_{r a d}=\frac{e^{2} E_{\omega}^{2}\left[1+2 \omega_{c} R e\left(\epsilon_{x}^{*} \epsilon_{y}\right) / \omega\right]}{2 m^{*}\left[\left(\omega-\omega_{c}\right)^{2}+\Gamma_{r a d}^{2}\right]},
$$

here $\mathcal{E}_{\mu}^{(0)}$ are the usual Landau energies, and the induced Floquet energy shift is given by the microwave energy $\mathcal{E}_{\text {rad }}$. The corresponding time-periodic Floquet modes in the $\left(P_{1}, P_{2}\right)$ representation are given by

$$
\Psi_{\mu, k}(P)=\exp \{-i \sin (2 \omega t) F(\omega)\} \phi_{\mu}\left(P_{1}\right) \delta\left(P_{2}-k\right),
$$

the index $k$ labels the degeneracy of the Landau-Floquet states, and $\phi_{\mu}\left(P_{1}\right)$ is the harmonic oscillator function in the $P_{1}$ representation

$$
\phi_{\mu}\left(P_{1}\right)=\left\langle P_{1} \mid \mu\right\rangle=\frac{1}{\sqrt{\pi^{1 / 2} 2^{\mu} \mu !}} e^{-P_{1}^{2} / 2} H_{\mu}\left(P_{1}\right),
$$

$H_{\mu}\left(P_{1}\right)$ is the Hermite polynomial and the function $F(\omega)$ is given as

$$
F(\omega)=\frac{\omega_{c}}{\omega}\left(\frac{e E_{\omega} l_{B}}{\omega^{2}-\omega_{c}^{2}}\right)^{2}\left[\omega^{2}-\omega_{c}^{2}+2 \omega^{2} \epsilon_{x}^{2}-2 \omega_{c}^{2} \epsilon_{y}^{2}+\frac{R e\left(\epsilon_{x}^{*} \epsilon_{y}\right)}{\omega \omega_{c}}\left(2 \omega^{4}-\omega^{2} \omega_{c}^{2}+\omega_{c}^{4}\right)\right] .(14)
$$

Let us now consider the complete Hamiltonian including the contribution from the periodic potential. When the transformation induced by $W(t)$ is applied, the Schrödinger equation in (1) becomes

$$
P_{0} \Psi^{(W)}=H_{0} \Psi^{(W)}+V_{W}(t) \Psi^{(W)},
$$

where $\Psi^{(W)}=W(t) \Psi$ and $V_{W}(t)=W(t) V(\mathbf{r}) W^{-1}(t)$. Notice that the periodic potential acquires a time dependence brought by the $W(t)$ transformation. The problem is now solved in the interaction representation using first order time dependent perturbation 
Photoconductivity in AC-driven modulated two dimensional electron gas in a perpendicular magnetic field 6 theory. In the interaction representation $\Psi_{I}^{(W)}=\exp \left\{i H_{0} t\right\} \Psi^{(W)}$, and the Schrödinger equation becomes

$$
i \partial_{t} \Psi_{I}^{(W)}=\left\{V_{W}(t)\right\}_{I} \Psi_{I}^{(W)} .
$$

The equation has the solution $\Psi_{I}^{(W)}(t)=U\left(t-t_{0}\right) \Psi_{I}^{(W)}\left(t_{0}\right)$, where $U(t)$ is the evolution operator. To first order in perturbation theory it is given by the expression

$$
U(t)=1-i \int_{-\infty}^{t} d t^{\prime}\left[W^{\dagger}\left(t^{\prime}\right) V(\mathbf{r}) W\left(t^{\prime}\right)\right]_{I} .
$$

The interaction is adiabatically turned off as $t_{0} \rightarrow-\infty$, in which case the asymptotic states are selected as the Landau-Floquet eigenvalues of $H_{0}$, i.e. $\left|\Psi_{I}^{(W)}\left(t_{0}\right)\right\rangle \rightarrow|\mu, k\rangle$. Utilizing the explicit expression for the $W$ transformation in (8) and after a lengthly calculation the matrix elements of the evolution operator can be worked out as

$$
\left\langle\mu, k|U(t)| \nu, k^{\prime}\right\rangle=\delta_{\mu \nu} \delta_{k k^{\prime}}-\sum_{l} \sum_{m n} \delta\left(k-k^{\prime}+l_{B} q_{n}^{(y)}\right) \frac{e^{i l_{B} q_{m}^{(x)}\left(k+l_{B} q_{n}^{(y)} / 2\right)} e^{i\left(\mathcal{E}_{\mu \nu}+\omega l\right) t}}{\mathcal{E}_{\mu \nu}+\omega l+\omega_{E}} C_{\mu \nu, m n}^{(l)},
$$

where $\omega_{E}=e l_{B}^{2}\left(q_{n}^{(y)} E_{x}^{c}-q_{m}^{(x)} E_{y}^{c}\right)$, and the explicit expression for $C_{\mu \nu, m n}^{(l)}$ is given by

$$
C_{\mu, \nu, m n}^{(l)}=l_{B}^{2} V_{m n} D_{\mu \nu}\left(\tilde{q}_{m n}\right)\left(\frac{\Delta_{m n}}{i\left|\Delta_{m n}\right|}\right)^{l} J_{l}\left(\left|\Delta_{m n}\right|\right) .
$$

In the previous expressions the discreet pseudomomentum are given as

$$
\begin{array}{ll}
q_{m}^{(x)}=2 \pi m / a, & q_{n}^{(y)}=2 \pi n / b, \\
\tilde{q}_{m n}=i l_{B}\left(q_{m}^{(x)}-i q_{n}^{(y)}\right) / \sqrt{2}, &
\end{array}
$$

$J_{l}$ denote the Legendre polynomials and $D_{\mu \nu}$ is given in terms of the generalized Laguerre polynomials according to

$$
D^{\nu \mu}(\tilde{q})=\langle\nu|D(\tilde{q})| \mu\rangle=e^{-\frac{1}{2}|\tilde{q}|^{2}} \begin{cases}\left(-\tilde{q}^{*}\right)^{\mu-\nu} \sqrt{\frac{\nu !}{\mu !}} L_{\nu}^{\mu-\nu}\left(|\tilde{q}|^{2}\right), & \mu>\nu, \\ \tilde{q}^{\nu-\mu} \sqrt{\frac{\mu !}{\nu !}} L_{\mu}^{\nu-\mu}\left(|\tilde{q}|^{2}\right), & \mu<\nu,\end{cases}
$$

and

$$
\Delta_{m n}=\frac{\omega_{c} l_{B}^{2} e E_{\omega}}{\omega\left(\omega^{2}-\omega_{c}^{2}+i \omega \Gamma_{r a d}\right)}\left[\omega\left(q_{m}^{(x)} e_{x}+q_{n}^{(y)} e_{y}\right)+i \omega_{c}\left(q_{m}^{(x)} e_{y}-q_{n}^{(y)} e_{x}\right)\right] .
$$

Summarizing, the solution to the original Schrödinger equation in Eq. (11) has been achieved by means os three successive transformations:

$$
\left|\Psi_{\mu, k}(t)\right\rangle=W^{\dagger} \exp \left\{-i H_{0} t\right\} U\left(t-t_{0}\right)|\mu, k\rangle,
$$

the explicitly expressions for $H_{0}, W$, and $U$ are given in Eqs. (17), (8), and (18) respectively. 


\section{Kubo formula for Floquet states.}

The usual Kubo formula for the conductivity must be modified in order to include the Floquet dynamics. In the presence of an additional $D C$ electric field the complete Hamiltonian is $H_{T}=H+V_{\text {ext }}$, where $H$ is the Hamiltonian in Eq. (11) and $V_{e x t}=$ $\frac{1}{m} \boldsymbol{\Pi} \cdot \mathbf{A}_{e x t}$, with $\mathbf{A}_{e x t}=\frac{\mathbf{E}_{0}}{\omega} \sin (\Omega t) \exp (-\eta|t|)$. The static limit is obtained with $\Omega \rightarrow 0$, and $\eta$ represents the rate at which the perturbation is turned on and off. In order to calculate the expectation value of the current density, we need the density matrix $\rho(t)$ which obeys the von Neumann equation

$$
i \hbar \frac{\partial \rho}{\partial t}=\left[H_{T}, \rho\right]=\left[H+V_{e x t}, \rho\right] .
$$

We write to first order $\rho=\rho_{0}+\Delta \rho$, where the leading term satisfies the equation

$$
i \hbar \frac{\partial \rho_{0}}{\partial t}=\left[H, \rho_{0}\right] \text {. }
$$

In agreement with Eq. (23), $\Delta \rho$ is transformed to

$$
\tilde{\Delta \rho}(t)=U_{I}^{\dagger}\left(t-t_{0}\right) \exp \left\{i H_{0} t\right\} W(t) \Delta \rho(t) W^{\dagger}(t) \exp \left\{-i H_{0} t\right\} U_{I}\left(t-t_{0}\right) .
$$

In terms of the transformed density matrix $\tilde{\Delta} \rho(t)$, Eq. (24) becomes

$$
i \hbar \frac{\partial \tilde{\Delta} \rho}{\partial t}=\left[\tilde{V}_{e x t}, \tilde{\rho}_{0}\right]
$$

where $\tilde{V}_{e x t}$ and $\tilde{\rho}_{0}$ are the external potential and quasi-equilibrium density matrix transformed in the same manner as $\tilde{\Delta} \rho$ in Eq. (26). The transformed quasi-equilibrium density matrix is assumed to have the form $\tilde{\rho}_{0}=\sum_{\mu}|\mu\rangle f\left(\mathcal{E}_{\mu}\right)\langle\mu|$, where $f\left(\mathcal{E}_{\mu}\right)$ is the usual Fermi function and $\mathcal{E}_{\mu}$ the Landau-Floquet levels. The justification for selecting a Fermi-Dirac distribution in the quasi-energy states is presented in the appendix (5.2). If we consider the region in which the conditions $\tau_{\omega} \ll \tau_{t r} \ll \tau_{\text {in }}$ hold, then the elastic and inelastic relaxation processes can be neglected as compared to the microwave field effects. The solution of the Boltzmann equation yields, a Fermi-Dirac distribution in the quasi-energy states [17, see appendix (5.2). It is straightforward to verify that this selection guarantee that the quasi-equilibrium condition in (25) is verified. Using the results in Eqs. (23) and (26), the expectation value of the density matrix can now be easily obtained from the integration of Eq. (27) with the initial condition $\Delta \rho(t) \rightarrow 0$ as $t \rightarrow-\infty$ giving for $t<0$

$$
\begin{aligned}
\left\langle\Psi_{\mu, k}|\Delta \rho(t)| \Psi_{\nu, k^{\prime}}\right\rangle & =\left\langle\mu, k|\tilde{\Delta} \rho(t)| \nu, k^{\prime}\right\rangle \\
& =\frac{e \mathbf{E}_{0}}{2} \cdot \int_{-\infty}^{t}\left[\frac{e^{i(\Omega-i \eta) t^{\prime}}}{\Omega} f_{\mu \nu}\left\langle\Psi_{\mu, k}\left|\Pi\left(t^{\prime}\right)\right| \Psi_{\nu, k^{\prime}}\right\rangle+(\Omega \rightarrow-\Omega)\right],
\end{aligned}
$$

where the definition $f_{\mu \nu}=f\left(\mathcal{E}_{\mu}\right)-f\left(\mathcal{E}_{\nu}\right)$ was used. The expectation value for the momentum operator is explicitly computed with the help of Eqs (8), (18), and (23), retaining terms linear in the modulation potential and after a lengthly calculations it yields

$$
\left\langle\Psi_{\mu k}\left|\Pi_{i}\right| \Psi_{\nu k^{\prime}}\right\rangle=\sqrt{e B} \sum_{l} \sum_{m n} \delta\left(k-k^{\prime}+l_{B} q_{n}^{(y)}\right) e^{i l_{B} q_{m}^{(x)}\left(k+l_{B} q_{n}^{(y)} / 2\right)} e^{i\left(\mathcal{E}_{\mu \nu}+\omega l-i \eta\right) t} \Delta_{\mu \nu, m n}^{(l)}(j) .
$$


Photoconductivity in AC-driven modulated two dimensional electron gas in a perpendicular magnetic field 8

Here the following definitions were introduced: $\mathcal{E}_{\mu \nu}=\mathcal{E}_{\mu}-\mathcal{E}_{\nu}, a_{j}=b_{j}=1$ if $j=x$, $a_{j}=-b_{j}=-i$ if $j=y$, and $\Delta_{\mu \nu, m n}^{(l)}(j)$ is given by

$$
\Delta_{\mu \nu, m n}^{(l)}(j)=-\frac{1}{\sqrt{2}}\left[\frac{a_{j} \tilde{q}_{m n}^{*} C_{\mu \nu, m n}^{(l)}}{\mathcal{E}_{\mu \nu}-\omega_{c}+\omega l-i \eta}+\frac{b_{j} \tilde{q}_{m n} C_{\mu \nu, m n}^{(l)}}{\mathcal{E}_{\mu \nu}+\omega_{c}+\omega l-i \eta}\right],
$$

the expression for $C_{\mu \nu, m n}^{(l)}$ is given in (19). It should be pointed out that in principle there is a zero order contribution (independent of $V$ ) to the expectation value of the momentum operator in (29); this would contribute to the direct cyclotron resonance heating that has a single peak around $\omega \sim \omega_{c}$. However this contribution has proved to be negligible [17, so it will be altogether omitted. Utilizing these results the time integral in Eq. (28) is readily carried out. The current density to first order in the external electric field can now be calculated from $\langle\mathbf{J}(t, \mathbf{r})\rangle=\operatorname{Tr}[\tilde{\Delta} \rho(t) \tilde{\mathbf{J}}(t)]$, the resulting expression represents the local density current. Here we are concerned with the macroscopic conductivity tensor that relates the spatially and time averaged current density $\mathbf{j}=\left(\tau_{\omega} \mathcal{A}\right)^{-1} \int_{0}^{\tau_{\omega}} d t \int d^{2} x\langle\mathbf{J}(t, \mathbf{r})\rangle$ to the averaged electric field; here $\mathcal{A}$ is the area of the system (it is understood that $\mathcal{A} \rightarrow \infty$ ). Assuming that the external electric field points along the $x$-axis the macroscopic conductivity can be worked out. The total conductivity is given by a sum $\sigma_{x i}=\sigma_{x i}^{D}+\sigma_{x i}^{(M M)}$; the dark conductivity is calculated when both the modulation and microwave radiation are switched-off as

$$
\sigma_{x i}^{D}=\frac{e^{2} \omega_{c}^{2}}{4 \hbar i} \sum_{\mu \nu}\left\{\frac{f_{\mu \nu}}{\Omega}\left[\frac{a_{i} \mu \delta_{\mu, \nu+1}}{\mathcal{E}_{\mu \nu}+\Omega-i \eta}+\frac{b_{i} \nu \delta_{\mu, \nu-1}}{\mathcal{E}_{\mu \nu}+\Omega-i \eta}\right]+(\Omega \rightarrow-\Omega)\right\},
$$

whereas the microwave-modulation (MM) induced conductivity is worked out as

$$
\sigma_{x i}^{(M M)}=\frac{e^{2} \omega_{c}^{2}}{4 \hbar i} \sum_{\mu \nu}\left\{\frac{f_{\mu \nu}}{\Omega} \sum_{m n} \sum_{l} \frac{\Delta_{\mu \nu, m n}^{(l)}(i) \Delta_{\nu \mu, m n}^{(-l)}(x)}{\mathcal{E}_{\mu \nu}+\omega l+\Omega-i \eta}+(\Omega \rightarrow-\Omega)\right\} .
$$

Selecting $i=x$ or $i=y$ the longitudinal and Hall conductivities can be selected. The denominators on the R.H.S. of the previous equations can be related to the advanced and retarded Green's functions $G_{\mu}^{ \pm}(\mathcal{E})=1 /\left(\mathcal{E}-\mathcal{E}_{\mu} \pm i \eta\right)$. To make further progress the real and absorptive parts of the Green's functions are separated taking the limit $\eta \rightarrow 0$ and using $\lim _{\eta \rightarrow 0} 1 /(\mathcal{E}-i \eta)=P 1 / \mathcal{E}+i \pi \delta(\mathcal{E})$, where $P$ indicates the principal-value integral. As usual the real and imaginary parts contribute to the Hall and longitudinal conductivities respectively. However, the previous expression would present a singular behavior that is an artifact of the $\eta \rightarrow 0$ limit. This problem is solved by including the disorder broadening effects. A formal procedure to obtain the Green function requires a self-consistent calculation using the Dyson equation for the self-energy with the magnetic and microwave fields, impurity, phonon, and other scattering effects included. A detailed calculation of $\operatorname{Im} G_{\mu}(\mathcal{E})$ incorporating all these elements is beyond the scope of the present work. Instead we choose a gaussian-type expression for the the density of states. This expression can be justified within a self-consistent Born calculation that incorporates the magnetic field and disorder effects [33, 34, 35, hence the density of 
Photoconductivity in AC-driven modulated two dimensional electron gas in a perpendicular magnetic field 9 states for the $\mu$-Landau level is represented as

$$
\operatorname{Im} G_{\mu}(\mathcal{E})=\sqrt{\frac{\pi}{2 \Gamma_{\mu}^{2}}} \exp \left[-\left(\mathcal{E}-\mathcal{E}_{\mu}\right)^{2} /\left(2 \Gamma_{\mu}^{2}\right)\right]
$$

with a broadening width given by

$$
\Gamma_{\mu}^{2}=\frac{2 \beta_{\mu} \hbar^{2} \omega_{c}}{\left(\pi \tau_{t r}\right)}
$$

the parameter $\beta_{\mu}$ takes into account the difference of the transport scattering time $\tau_{t r}$ determining the mobility $\mu$, from the single-particle lifetime $\tau_{s}$. In the case of shortrange scatterers $\tau_{t r}=\tau_{s}$ and $\beta_{\mu}=1$. An expression for $\beta_{\mu}$, suitable for numerical evaluation, that applies for a long-range screened potential is given in reference [17]; $\beta_{\mu}$ decreases for higher Landau levels; e.g. $\beta_{0} \approx 50, \beta_{50} \approx 10.5$.

The static limit with respect to the external field is obtained taking $\Omega \rightarrow 0$ in Eqs. (31) and (32). In what follows results are presented for the microwave-modulation (MM) induced longitudinal conductivity, the dark conductivities as well as the MM Hall conductivity are quoted in the appendix. Hence the MM induced longitudinal conductance is worked out as

$\sigma_{x x}^{(M M)}=\frac{e^{2} l_{B}^{2}}{\pi \hbar} \int d \mathcal{E} \sum_{\mu \nu} \sum_{l} \sum_{m n} \operatorname{Im}_{\mu}(\mathcal{E}) B^{(l)}\left(\mathcal{E}, \mathcal{E}_{\nu}\right)\left|q_{n}^{(y)} J_{l}\left(\left|\Delta_{m n}\right|\right) V_{m n} D_{\mu \nu}\left(\tilde{q}_{m n}\right)\right|^{2}$,

where the following function has been defined

$$
B^{(l)}\left(\mathcal{E}, \mathcal{E}_{\nu}\right)=-\left.\frac{d}{d \mathcal{E}_{0}}\left\{\left[f\left(\mathcal{E}+l \omega+\omega_{E}+\mathcal{E}_{0}\right)-f(\mathcal{E})\right] \operatorname{Im} G_{\nu}\left(\mathcal{E}+l \omega+\omega_{E}+\mathcal{E}_{0}\right)\right\}\right|_{\mathcal{E}_{0}=0} .(36)
$$

Notice that $\sigma_{x x}^{(M M)}$ contains a contribution $\sigma_{x x}^{(M)}$ that depends only on the modulation potential, it can be extracted from Eq. (35) if the microwave field is switched-off.

As usual the resistivities are obtained from the expression $\rho_{x x}=\sigma_{x x} /\left(\sigma_{x x}^{2}+\sigma_{x y}^{2}\right)$ and $\rho_{x y}=\sigma_{x y} /\left(\sigma_{x x}^{2}+\sigma_{x y}^{2}\right)$. The relation $\sigma_{x y} \gg \sigma_{x x}$ holds in general, hence it follows that $\rho_{x x} \propto \sigma_{x x}$, and the longitudinal resistivity follows the same oscillation pattern as that of $\sigma_{x x}$.

\section{Results.}

The expression in Eq. (35) can be numerically evaluated after the Fourier components $V_{m n}$ of the periodic potential are specified. We shall consider a square lattice potential of the form

$$
V(\mathbf{r})=V_{0}\left[\cos \left(\frac{2 \pi x}{a}\right)+\cos \left(\frac{2 \pi y}{a}\right)\right] .
$$

In our calculations it is assumed that a superlattice is cleaved at the interface of an ultraclean $G a A s / A l_{x} G a_{1-x} A s$ heterostructure with high electron mobility, $\mu \sim$ $0.5-2.5 \times 10^{7} \mathrm{~cm}^{2} / V s$; the periodic potential has the form given in (37) with parameters $a \sim 20-200 \mathrm{~nm}$ and $V_{0}=0.05 \mathrm{meV}$. The other parameters of the sample are estimated as effective electron mass $m^{*}=0.067 m_{e}$, fermi energy $\epsilon_{F}=10 \mathrm{meV}$, electron density 
Photoconductivity in AC-driven modulated two dimensional electron gas in a perpendicular magnetic field 10

$n=3 \times 10^{11} \mathrm{~cm}^{-2}$, and temperature $T=1 \mathrm{~K}$. For the applied external fields we consider: magnetic fields in the range $0.05-0.4$ Tesla, and microwave radiation with frequencies $f \sim 10-200 G h z$; and field intensity $\left|\vec{E}_{\omega}\right| \sim 1-100 \mathrm{~V} / \mathrm{cm}$, that corresponds to a microwave power characterized by the dimensionless quantity $\alpha \sim c \epsilon_{0}\left|E_{\omega}\right|^{2} /\left(m^{*} \omega^{3}\right)$ that varies in the range $\alpha \sim 0.01-2$. The relaxation time $\tau_{t r}$ in Eq. (33) is related with the zero field electron mobility through $\mu=e \tau_{t r} / m^{*}$, and $\beta_{\mu} \approx 10.5$, a value that is justified for large filling factors $\mu \approx 50$ [17]. A detailed account of the electron dynamics requires to distinguish between various time life's; following reference [37, $\Gamma_{\text {rad }}$ in Eq. (10) is related to the radiative decay width that is interpreted as coherent dipole re-radiation of electromagnetic waves by the oscillating $2 \mathrm{D}$ electrons excited by microwaves, it is given by $\Gamma_{\text {rad }}=2 \pi^{2} \hbar n e^{2} /\left(3 \epsilon_{0} c m^{*}\right)$. In all the examples, except in Fig. 8. we consider the linear regime, the dc-electric field is included only through the Kubo formula, hence $\omega_{E}=0$ in Eqs. (35), (36). In the case of Fig. 8 the non-linear dc-electric field effects are included using the solution to the classical equations of motion with both ac- and dc-electric fields (10).

Plots of the longitudinal and Hall resistivities as a function of the magnetic field intensity are displayed in Fig. 1 The total longitudinal resistance shows a strong oscillatory behavior with distinctive NRS, this behavior is contrasted with the dark contributions that presents only the expected Shubnikov-de-Hass oscillations. The total Hall resistance presents a monotonous behavior, yet perceptible microwave induced oscillations in the Hall effect can be observed if one considers $\Delta \rho_{x y}=\rho_{x y}-\rho_{x y}^{d a r k}$, see the inset.

Fig. 22 displays plots of the total longitudinal conductivity as a function of $\epsilon=\omega / \omega_{c}$. $\sigma_{x x}$ shows a strong oscillatory behavior, with distinctive negative conductance states. The periodicity as well as the number of NCS depend on the intensity of the microwave radiation. For weak microwave intensity $(\alpha=0.01), \sigma_{x x}$ is positive with a moderate oscillatory behavior. As the microwave intensity increases $(\alpha=0.1)$, strong oscillations in $\sigma_{x x}$ are observed with minima centered at $\epsilon \sim 1.2, \epsilon \sim 2.2, \epsilon \sim 3.2$, and $\epsilon \sim 4.2$; only the two last minima correspond to NCS. A further increase in the microwave intensity ( $\alpha=0.4$ ) yields several NCS. In the region $\epsilon \leq 3$ the oscillation period is reduced to $\epsilon=\frac{1}{2}$. In general it is observed that $\sigma_{x x}$ vanishes at $\omega / \omega_{c}=j$ for $j$ integer. The oscillations follow a pattern with minima centered at $\omega / \omega_{c}=j+\frac{1}{2}(l-1)+\delta$, and maxima centered at $\omega / \omega_{c}=j+\frac{1}{2}(l-1)-\delta$, where $j=1,2,3 \ldots \ldots ., \delta \approx 1 / 5$, and $l$ is the dominant multipole that contributes to the conductivity in Eq. (35) . For moderate microwave power the $l=1$ "one photon" stimulated processes dominate, corresponding to what is observed for $\alpha=0.1$. For $\alpha=0.4$ the results can be interpreted as the results of "one and two photon" processes $(l=1$ and $l=2)$. To understand the origin of NCS it is noticed from Eq. (35) that, for small microwave power, $\sigma_{x x}^{\omega}$ is dominated by the $l=0$ Bessel term, that is always positive. Negative conductance states arise when the $l=1$ and $l=0$ terms become comparable: $\left|J_{0}(|\Delta|)\right|^{2} B^{(0)} \sim\left|J_{1}(|\Delta|)\right|^{2} B^{(1)}$. A simple analysis show that this condition is fulfilled for $|\Delta| \sim 0.1$. Using the expression in Eq. (22), the condition to produce NCS can be estimated as $\left|\mathbf{E}_{\omega}\right|>E_{t h}$ where $E_{t h} \approx 0.1 a \Gamma_{\text {rad }} / \sqrt{8} e l_{B}$. 
For the parameter used in Fig. 2. $E_{t h} \approx 10 \mathrm{~V} / \mathrm{cm}$ or $\alpha_{t h} \approx 0.15$, in good agreement with the results displayed by the plots.

Next we consider the dependence of $\rho_{x x}$ on the lattice parameter $a$. Plots of $\rho_{x x}$ versus $\epsilon=\omega / \omega_{c}$ for various selections of $a$ are presented in (Fig. 31). NRS appear only in a narrow window of values of $a$ around $a^{*}$, for which the oscillations amplitude of $\rho_{x x}$ attains its maximum. In the present case: $a^{*} \approx 25 \mathrm{~nm}$. This behavior follows from the structure of the MM induced longitudinal conductance $\sigma_{x x}^{M M}$ given in (35). Taking into account the form of $D_{\mu \nu}\left(\tilde{q}_{m n}\right)$ in Eq. (21) , the leading dependence of $\sigma_{x x}^{M M}$ on the lattice parameter is given approximately by $\sigma_{x x}^{M M} \sim \frac{1}{a^{4}} \exp \left(-|\tilde{q}|^{2} / 2\right)$. According to Eq. (20) $\tilde{q} \propto 1 / a$, consequently the MM contribution is significant only when $a$ is near to $a^{*}$, that is determined as: $a^{*} \sim \pi l_{B} / \sqrt{2}$. This estimation is in very good agreement with the numerical results presented in the plots.

Negative magnetoresistance requires ultra-clean samples, the phenomenon appears when the electron mobility exceeds a threshold $\mu_{t h}$. Fig. 4 displays $\rho_{x x}$ v.s. $\omega / \omega_{c}$ plots for three selected values of $\mu$. For $\mu \approx 0.5 \times 10^{7} \mathrm{~cm}^{2} / V s$ an almost linear behavior $\rho_{x x}$ is clearly depicted (except in the Shubnikov-deHass region). As the electron mobility increases to $\mu \approx 1.5 \times 10^{7} \mathrm{~cm}^{2} / V s$, the resistance oscillations are clearly observed; however, several NRS only appear when the mobility is increased to $\mu \approx 2.5 \times 10^{7} \mathrm{~cm}^{2} / V s$. Eqs. (35136) contain the main ingredients that explain the huge increase observed in the longitudinal conductance (and resistance), when the periodically modulated system is irradiated by microwaves and its critical dependence on the electron mobility. In the standard expression for the Kubo formula there are no Floquet replica contribution, hence $\omega$ can be set to zero in (36), if that is the case $B^{(l)}$ becomes proportional to the energy derivative of the Fermi distribution, that in the $T \rightarrow 0$ limit becomes of the form $\delta\left(\mathcal{E}-\mathcal{E}_{F}\right)$, and the conductivity is positive definite depending only on those states lying at the Fermi level. On the other hand, as a result of the periodic structure induced by the microwave radiation, $B^{(l)}$ contains a second contribution proportional to the derivative of the density of states: $\frac{d}{d \mathcal{E}} \operatorname{Im} G_{\nu}(\mathcal{E}+l \omega)$. Due to the oscillatory structure of the density of states, this extra contribution takes both positive and negative values. According to Eq. (33) this second term (as compared to the first one) is proportional to the electron mobility, hence for sufficiently high mobility the new contribution dominates leading to negative conductance states.

The model is used to test chirality effects induced by the magnetic field. Fig. [5 shows $\sigma_{x x}$ v.s. $\omega / \omega_{c}$ plots for various $\mathbf{E}_{\omega}$ field polarization's with respect to the current. It is observed that the amplitude of the $\sigma_{x x}$ oscillations are bigger for linear transverse polarization as compared to the longitudinal polarization case. Similarly, the oscillation amplitudes are enhanced for negative circular polarization as compared to the positive circular polarization results; in particular NCS are observed only for negative circular polarization. These results are understood recalling that for negative circular polarization and $\omega \approx \omega_{c}$ the electric field rotates in phase with respect to the electron cyclotron rotation. 
Fig. [6 illustrates the fact that the strong oscillations in $\sigma_{x x}$ originates from the combined microwave-modulation effects. The dark contribution, Eq. (38), shows the expected $\sigma_{x x}^{D} \propto B$ linear behavior. The contribution arising solely from the periodic modulation $\sigma_{x x}^{M}$ can be isolated from Eq. (35) by switching-off the microwave field, the dashed line shows a smooth behavior. The combined microwave-modulation contribution is obtained from $\sigma_{x x}^{M M}-\sigma_{x x}^{M}$; the dotted line clearly shows that this contribution includes the main oscillatory behavior of the full $\sigma_{x x}$, furthermore it is the only contribution that becomes negative. Hence, it is concluded that both the periodic modulation and the microwave radiation are essential in order to observe the strong $\sigma_{x x}$ oscillations and the NCS.

Next we explore the behavior of the longitudinal conductivity as a function of the microwave radiation intensity. As the intensity of the electric microwave field $\left(E_{\omega}\right)$ is increased higher multipole $(l)$ terms needs to be evaluated; in the explored regime convergent results are obtained including terms up to the $l=5$ multipole. In Fig. 7 results are presented for $\sigma_{x x} v s E_{\omega}$; the selected values of $\epsilon=\omega / \omega_{c}$ correspond to minima or maxima of $\sigma_{x x}$ in figure 2. In general it is observed that for values corresponding to maxima i.e. $\epsilon=j-\delta ; \quad j=1,2,3 \ldots, \sigma_{x x}$ remains positive for all microwave field intensities. On the other hand, the minima corresponding to $\epsilon=j+\delta ; \quad j=1,2,3 \ldots$ are related to NCS around a region $\left|E_{\omega}\right| \in[10,25] \mathrm{V} / \mathrm{cm}$. Increasing the microwave intensity leads to the disappearance of the NCS, except for the first minima $(\epsilon \sim 1.1)$.

The non-linear regime with respect to the dc-external field can also be explored within the present formalism. The effect is included using the solution to the classical equations of motion with both ac- and dc-electric fields, Eq. (10). A possible connection between the observed $Z R S$ in $G a A s / A l_{x} G a_{1-x} A s$ heterostructures [1, 3, 2, 4] and the the predicted NRS 10, 11, 12, 13, 14, 15, 16, 17] was put forward by Andreev etal. 13], noting that a general analysis of Maxwell equations shows that $N R S$ induces an instability that drives the system into a ZRS. This mechanism requires the longitudinal current $j_{x x}$ as a function of $E_{d c}$ to have a single minimum, the system instability will evolve to the value $E_{d c}$ in which $j_{x x}$ cancel. Returning to the irradiated superlattice case, in Fig. 8 it is observed that in general the $j_{x x} v s . E_{d c}$ plot has an oscillatory behavior, with more than one minima. Hence the conditions of the Andreeev mechanism do not apply. Consequently, negative conductance states may be probably observed in 2-dimensional superlattices, when exposed to both magnetic and microwave fields.

\section{Conclusions.}

We have considered a model to describe the microwave photoconductivity of a 2DES in the presence of a magnetic field, and a $2 \mathrm{D}$ periodic modulation. We presented a thoroughly discussion of the method that takes into account the Landau and microwave contributions in a non-perturbative exact way, the periodic potential effects are treated perturbatively. The formalism exploits the symmetries of the problem: the exact solution of the Landau-microwave dynamics (77) is obtained in terms of the electric- 
magnetic generators (5) as well as the solutions to the classical equations of motion (92). The spectrum and Floquet modes are explicitly worked out (11). In our model, the Landau-Floquet states act coherently with respect to the oscillating field of the superlattice potential, that in turn induces transitions between these levels. Based on this formalism, a Kubo-like formula is provided (35) that consistently takes into account the oscillatory Floquet structure of the problem.

It is found that both $\sigma_{x x}$ and $\rho_{x x}$ exhibit strong oscillations governed by $\epsilon=\omega / \omega_{c}$. The oscillations follow a pattern with minima centered at $\omega / \omega_{c}=j+\frac{1}{2}(l-1)+\delta$, and maxima centered at $\omega / \omega_{c}=j+\frac{1}{2}(l-1)-\delta$, where $j=1,2,3 \ldots \ldots ., \delta \approx 1 / 5$ and $l$ is the dominant multipole contribution. NRS develop for sufficiently strong microwave power (Fig. (2)), in a narrow window of values of the lattice parameter $\left(a \sim l_{B}\right)$ (Fig. (3)), and for high electron mobility samples (Fig. 4). The explanation for the NRS can be traced down to Eqs. (35) and (36); the longitudinal photoconductivity contains a new contribution proportional to the derivative of the density of states: $\frac{d}{d \mathcal{E}} \operatorname{Im} G_{\nu}(\mathcal{E}+l \omega)$. Due to the oscillatory structure of the density of states this extra contribution takes both positive and negative values. This term is proportional to the electron mobility, hence for sufficiently high mobility the new contribution dominates leading to negative conductivity states. Unlike the semiclassical origin of magnetoresistance oscillations observed in an antidot array for commensurate values of the ratio $R_{c} / a$ [25], these conductance oscillations have a quantum origin and would only appear in a narrow window of values of $a$, around $a \sim l_{B}$.

In conclusion, it is proposed that the combined effect of: periodic modulation, perpendicular magnetic field, plus microwave irradiation of 2DES give rise to interesting oscillatory conductance phenomena, with the possible development of NCS and NRS. One should stress that according to our results, the production of NRS requires ultraclean samples with electron mobilities of order $\mu \approx 2.5 \times 10^{7} \mathrm{~cm}^{2} / V s$ (see Fig. (4). The electron mobilities in the fabricated arrays of periodic scatterers so far [24, 25] are $\mu \approx 2.5 \times 10^{6} \mathrm{~cm}^{2} / \mathrm{Vs}$, consequently an increase on the electron mobilities of these kind of experimental setups by an order of magnitude would be required in order to observe the phenomena described in this work.

\subsection{Appendix A: Dark and Hall conductivities.}

In section (31) it was explained in detail the method to obtain the final expression for the modulation-microwave induced conductance Eq. (35). Working along a similar procedure the expression for the remaining conductivities are worked from equations (31) and (32). First we quote the longitudinal dark conductance

$$
\sigma_{x x}^{D}=\frac{e^{2} \omega_{c}^{2}}{\pi \hbar} \sum_{\mu} \mu \int d \mathcal{E} \operatorname{Im} G_{\mu}(\mathcal{E}) \frac{d f}{d \mathcal{E}} \operatorname{Im} G_{\mu}\left(\mathcal{E}+\omega_{c}\right)
$$

whereas the Hall conductance reads

$$
\sigma_{x y}^{D}=\frac{e^{2} \omega_{c}^{2}}{\pi \hbar} \sum_{\mu} \mu \int d \mathcal{E} \operatorname{Im} G_{\mu}(\mathcal{E})\left[f\left(\mathcal{E}_{\mu}-\omega_{c}\right)-f(\mathcal{E})\right] P \frac{1}{\left(\mathcal{E}-\mathcal{E}_{\mu}+\omega_{c}\right)^{2}}
$$


Photoconductivity in AC-driven modulated two dimensional electron gas in a perpendicular magnetic field 14

where $P$ indicates the principal-value integral. The final result for the MM assisted longitudinal conductivity was quoted in Eq. (35)). The MM induced Hal conductivity is calculated to give

$$
\sigma_{x y}^{(M M)}=\frac{e^{2} l_{B}^{4}}{\pi \hbar} \int d \mathcal{E} \sum_{\mu \nu} \sum_{l} \sum_{m n} \operatorname{Im} G_{\mu}(\mathcal{E})\left[f\left(\mathcal{E}_{\nu}\right)-f(\mathcal{E})\right] T_{m n}^{l}\left|J_{l}\left(\left|\Delta_{m n}\right|\right) V_{m n} D_{\mu \nu}\left(\tilde{q}_{m n}\right)\right|^{2},
$$

were the function $T_{m n}^{l}$ is defined as

$$
T_{m n}^{l}=\omega_{c}^{3} \frac{\left(q_{m}^{(x)}\right)^{2}+\left(q_{n}^{(y)}\right)^{2}}{\left(\mathcal{E}+\omega l-\mathcal{E}_{\nu}\right)\left|\left(\mathcal{E}+\omega l-\mathcal{E}_{\nu}\right)^{2}-\omega_{c}^{2}\right|^{2}} .
$$

\subsection{Appendix B: Microwave-driven distribution function.}

Within the time relaxation approximation the Boltzmann equation can be written as

$$
\frac{\partial f}{\partial t}+\frac{\partial f}{\partial \mathbf{p}} \cdot(e \mathbf{E}+e \mathbf{v} \times \mathbf{B})=-\frac{f-f_{F}}{\tau_{t r}}-\frac{f-f_{F}}{\tau_{i n}},
$$

where $f_{F}$ is the Fermi-Dirac distribution and we distinguish between the elastic rate $\tau_{t r}^{-1}$ and inelastic or energy relaxation rate $\tau_{i n}^{-1}$. As it already mentioned, we assume the validity of the following conditions: $\tau_{\omega} \ll \tau_{t r} \ll \tau_{i n}$, and certainly the inelastic processes can be safely ignored. Furthermore, due to the $a c$-electric field (3), the L.H. S. of the previous equation is estimated to be of order $f / \tau_{\omega}$; hence, in a first approximation the elastic scattering contribution can also be neglected. The resulting Vlasov equation has the exact solution $f(\mathbf{p}, t)=f_{F}\left(\mathbf{p}-m^{*} \mathbf{v}(t)\right)$, where the velocity $\mathbf{v}(t) \equiv\left(\dot{\eta}_{1}, \dot{\zeta}_{1}\right)$ solves exactly the same classical equations of motion that follow from (9), and the initial condition is selected as, $f \rightarrow f_{F}$ as the external electric field is switched-off. In particular it is verified that $m^{*}|\mathbf{v}(t)|^{2} / 2=\mathcal{E}_{\text {rad }}$ coincides with the Floquet energy shift produced by the microwave radiation (11). The steady-state distribution, evaluated at the Landau energy $\mathcal{E}=\mathcal{E}_{\mu}^{(0)}$, is obtained by averaging $f_{F}\left(\mathbf{p}-m^{*} \mathbf{v}(t)\right)$ over the oscillatory period

$$
\left\langle f_{F}\right\rangle=\frac{1}{\tau_{\omega}} \int_{0}^{\tau_{\omega}} f_{F}\left(\mathcal{E}_{\mu}^{(0)}+\mathcal{E}_{\text {rad }}+2 \cos \omega_{c} t \sqrt{\mathcal{E}_{\mu}^{(0)} \mathcal{E}_{\text {rad }}}\right) d t .
$$

In general it is verified that $\mathcal{E}_{\text {rad }} \ll \mathcal{E}_{\mu}^{(0)}$, thus expanding to first order one finds $\left\langle f_{F}\right\rangle \approx f_{F}\left(\mathcal{E}_{\mu}^{(0)}+\mathcal{E}_{\text {rad }}\right)=f_{F}\left(\mathcal{E}_{\mu}\right)$. Hence, it is verified that a rapid relaxation of the Fermi distribution to the quasi-energy states is a reasonable assumption. The arguments presented in this appendix have been introduced by Mikhailov [37] in order to explore the possibility that the microwave radiation leads to a population inversion; however, it is concluded that it would require a rather high microwave intensity $\mathcal{E}_{\text {rad }}>\mathcal{E}_{F}$.

\section{Acknowledgments}

We acknowledge the partial financial support endowed by CONACyT through grants No. 42026-F and G32736-E, and UNAM project No. IN113305. 
Photoconductivity in AC-driven modulated two dimensional electron gas in a perpendicular magnetic field 15

\section{References}

[1] M. A. Zudov, R. R. Du, J. A. Simmons, J. L. Reno, Phys. Rev. B 64 (2001) 201311(R).

[2] M. A. Zudov, R. R. Du, L. N. Pfeiffer, K. W. West, Phys. Rev. Lett 90 (2003) 046807.

[3] R. G. Mani, J. H. Smet, K. von klitzing, V. Narayanamurti, W.b. Johnson, Umansky, Nature 420 (2002) 646; Phys. Rev. Lett 92 (2004) 146801.

[4] R. G. Mani, Physica E 22 (2004) 1.

[5] S. I. Dorozhkin, JETP. Letters. 77 (2003) 577.

[6] R. L. Willett, L. N. Pfeiffer, K. W. West, Phys. Rev. Lett 93 (2004) 026804.

[7] M. A. Zudov, Phys. Rev. B 69 (2004) 041304(R).

[8] A. E. Kovalev, S. A. Zvyagin, C. R. Bowers, J. L. Reno, J. A. Simmons, Solid State Commun. $1130(2004) 379$.

[9] S. A. Studenikin, M. Potemski, A. Sachrajda, M. Hilke, L. N. Pfeiffer, K. W. West, cond-mat/0404411 (2004).

[10] V. I. Ryzhii, Sov. Phys. Solid State 11 (1970) 2078.

[11] V. I. Ryzhii, R. Suris, J Phys. Cond. Matt. 15 (2003) 6855.

[12] A. C. Durst, S. Sachdev, N. Read, S. M. Girvin, Phys. Rev. Lett. 91 (2003) 086803.

[13] A. V. Andreev, I. L. Aleiner, A. J. Millis, Phys. Rev. Lett. 91 (2003) 056803.

[14] J. Shi, X. C. Xie, Phys. Rev. Lett. 91 (2003) 086801.

[15] X. L. Lei, S. Y. Liu, Phys. Rev. Lett. 91 (2003) 226805.

[16] M. G. Vavilov, I. L. Aleiner, Phys. Rev. B 69 (2004) 035303.

[17] M. Torres, A. Kunold, Phys. Rev. B 71 (2005) 115313-1.

[18] M. Torres, A. Kunold, Phys. Stat. Sol. b 71 (2005) 1192.

[19] I. A. Dmitriev, A. D. Mirlin, D. G. Polyakov, Phys. Rev. Lett. 91 (2003) 226802.

[20] I. A. Dmitriev, M. G. Vavilov, I. L. Aleiner, A. D. Mirlin, D. G. Polyakov, Phys. Rev. B 71 (2005) 115316.

[21] J. P. Robinson, M. P. Kennett, , N. R. Cooper, V. I. Fal'ko, Phys. Rev. Lett. 93 (2004) 036804

[22] D. Weiss, K. v. Klitzing, K. Ploog, G. Weimann, Europhys. Lett. 8 (1989) 179; R. W. Winkler, J. P. Kotthaus, K. Ploog, Phys. Rev. Lett. 62 (1989) 1177; D. Weiss, M. L. Roukes, A. Menschig, P. Grambow, K. v. Klitzing, G. Weimann, Phys. Rev. Lett. . 66 (1991) 2790.

[23] For a review see R. Schuster, K. Ensslin, Adv. Solid State Phys. 34 (1994) 195.

[24] C. Albrecht, J. H. Smet, K. v. Klitzing, D. Weiss, V. Umansky, H. Schweizer, Phys. Rev. Lett. 86 (2001) 147.

[25] E. Vasiliadou, R Fleischmann, D. Weiss, D. Heitmann, K. v. Klitzing, T. Geisel , R. Bergmann, H. Schweizer, C. T. Foxon, Phys. Rev. B 52 (1995) R8658.

[26] J. Dietel, L. I. Glazman, F. W. J. Hekking, F. von Oppen, Phys. Rev. B 71 (2005) 045329.

[27] G. Gumbs Phys. Rev. B 72 (2005) 125342.

[28] K. Husimi, Prog. Theor. Phys. 9 (1953) 381.

[29] A. Kunold, M. Torres, Phys. Rev. B 61 (2000) 9879.

[30] M. Torres, A. Kunold, Phys. Lett. A 323 (2004) 2890.

[31] N. Ashby and S.C. Miller, Phys. Rev. B 139 (1965) A428.

[32] A. Kunold, M. Torres, Annals of Physics 315 (2005) 532.

[33] T. Ando, Y. Uemura, J. Phys. Soc. Japan. 36 (1974) 959.

[34] R. R. Gerhardts, Z. Phys. B 21 (1975) 275; Ibid. 21 (1975) 285.

[35] T. Ando, A. B. Fowler, F. Stern, Rev. Mod. Phys. 54 (1982) 437.

[36] T. Dittrich, P. hanggi, G.L. Ingold, B. Kramer, G. Schon, W. Zwerger, "Quantum transport and dissipation", Wiley-VCH (1998).

[37] S. A. Mikhailov, Phys. Rev. B 70 (2004) 165311. 
Photoconductivity in AC-driven modulated two dimensional electron gas in a perpendicular magnetic field 16

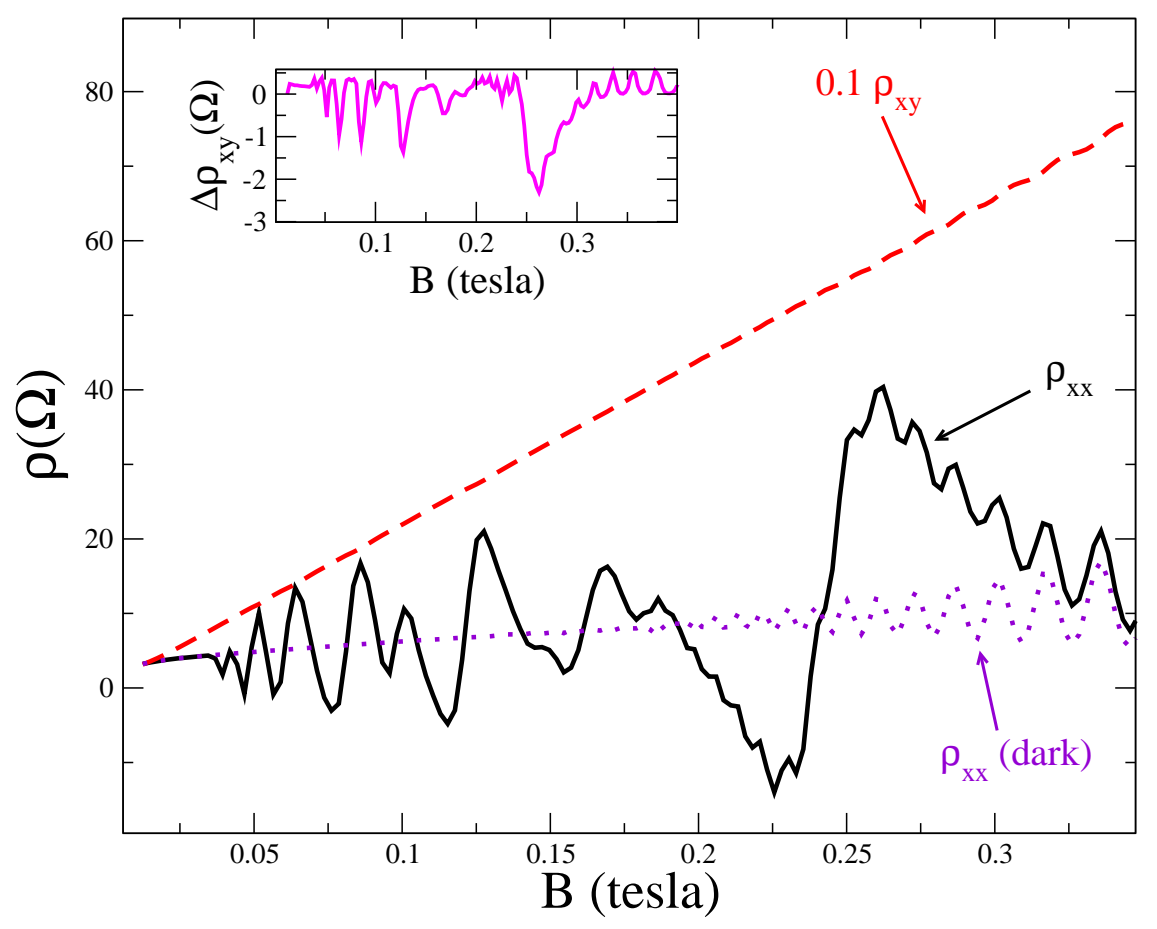

Figure 1. Magnetoresistivity $\rho_{x x}$ versus $B$ without radiation and modulation (dotted line) and under microwave radiation plus periodic modulation (solid line). The dashed line corresponds to the total Hall resistance $\rho_{x y}$, whereas the inset shows the results for the subtracted Hall resistance $\Delta \rho_{x y}=\rho_{x y}-\rho_{x y}^{\text {dark }}$. The microwave polarization is linear transverse (with respect to the current), with $f=100 G h z$ and $\alpha=c \epsilon_{0}\left|E_{\omega}\right|^{2} /\left(m^{*} \omega^{3}\right)$ $=0.4$. The other parameters are selected as follows: $a=25 \mathrm{~nm}, V_{0}=0.05 \mathrm{meV}$, $m^{*}=0.067 m_{e}, \mu \approx 2.5 \times 10^{7} \mathrm{~cm}^{2} / V s, \epsilon_{F}=10 \mathrm{meV}, T=1 \mathrm{~K}$. 
Photoconductivity in AC-driven modulated two dimensional electron gas in a perpendicular magnetic field 17

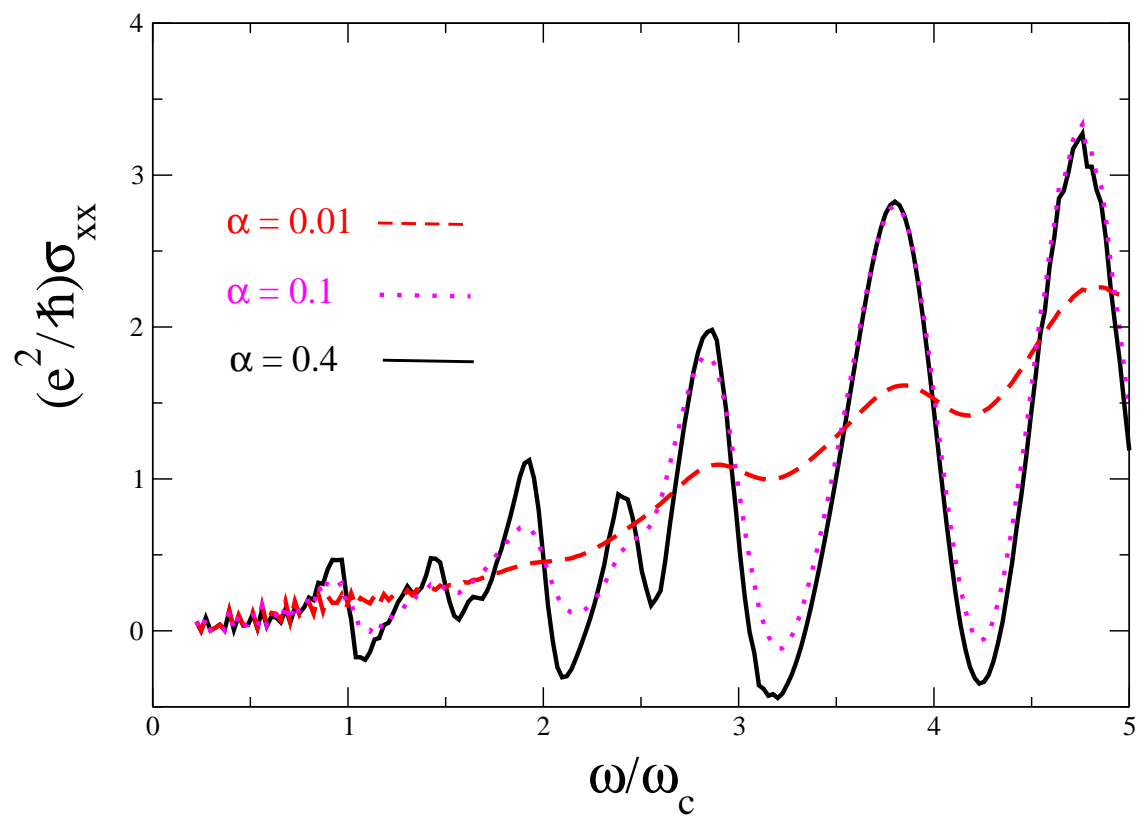

Figure 2. Longitudinal conductivity versus $\epsilon=\omega / \omega_{c}$ for three values of the microwave power intensity: $\alpha=0.01$ dashed line, $\alpha=0.1$ dotted line, and $\alpha=0.4$ continuos line. The other parameters have the same values as in Fig. 1

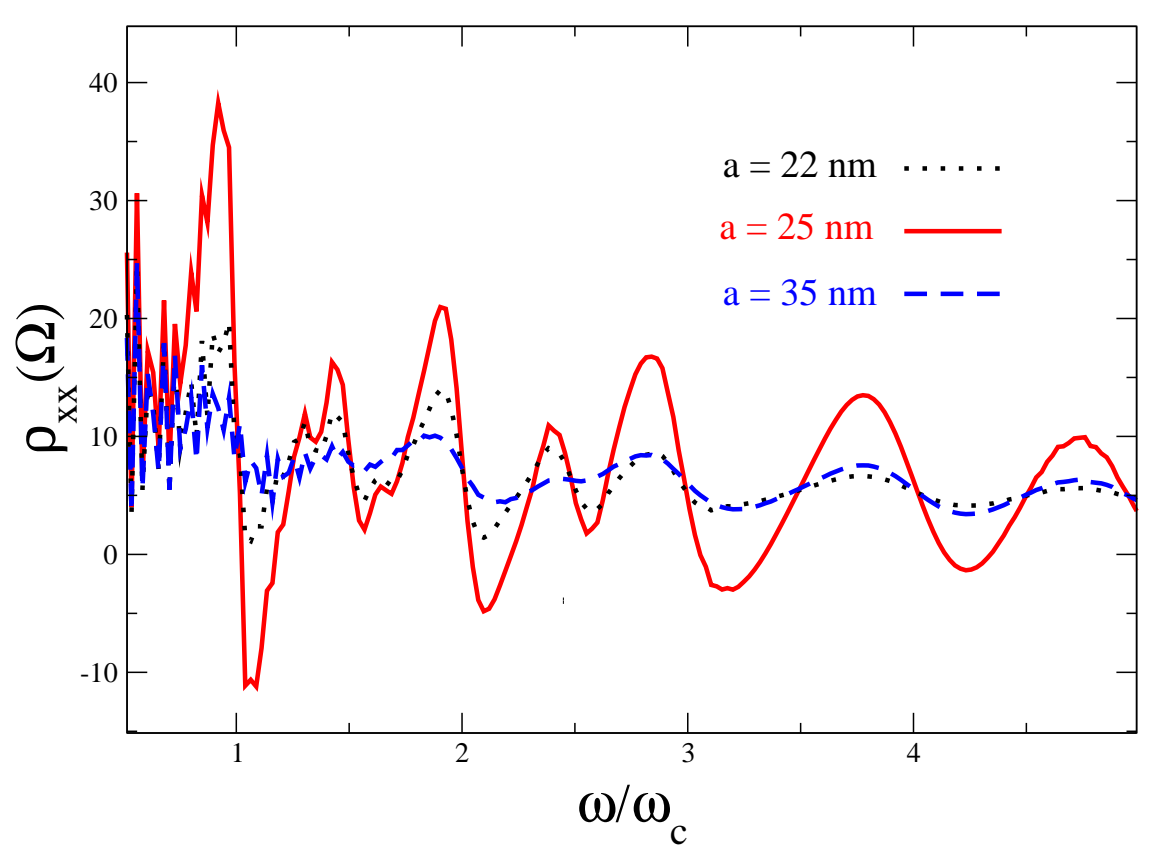

Figure 3. Longitudinal resistivity versus $\epsilon=\omega / \omega_{c}$ for three values of the lattice parameter: $a=22 \mathrm{~nm}$ dotted line, $a=25 \mathrm{~nm}$ continuos line, and $a=35 \mathrm{~nm}$ dashed line. The microwave intensity is $\alpha=0.4$ and the other parameters have the same values as in Fig. 1] 
Photoconductivity in AC-driven modulated two dimensional electron gas in a perpendicular magnetic field 18

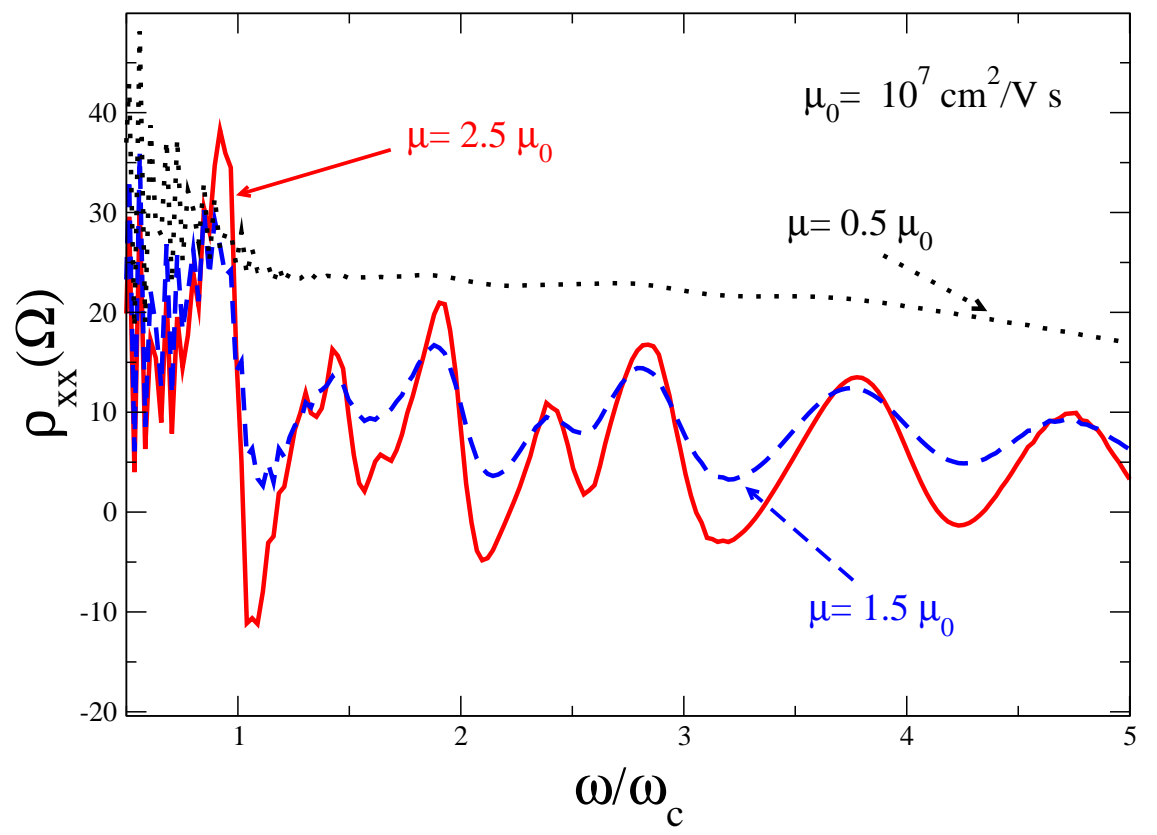

Figure 4. Longitudinal resistivity as a function $\epsilon=\omega / \omega_{c}$ for three values of the electron mobility: $\mu=0.5 \times 10^{7} \mathrm{~cm}^{2} / V s$ dotted line, $\mu=1.5 \times 10^{7} \mathrm{~cm}^{2} / V s$ dashed line, and $\mu=2.5 \times 10^{7} \mathrm{~cm}^{2} / V s$ continuos line. The microwave power is given by $\alpha=0.4$, the other parameters have the same values as in Fig. 1.
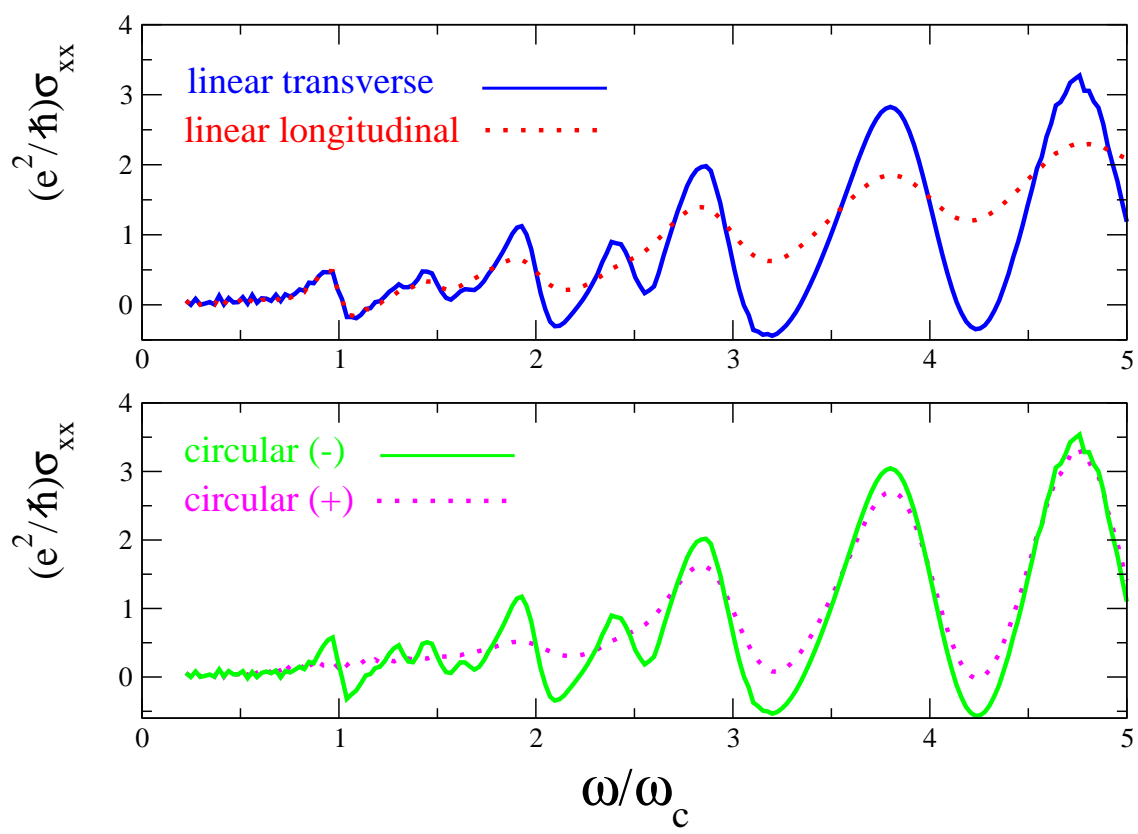

Figure 5. Longitudinal conductance vs. $\epsilon=\omega / \omega_{c}$ for various microwave $E_{\omega}$-field polarization's with respect to the current. In figure $(a)$ the continuos and dotted lines correspond to linear transverse and longitudinal polarization's respectively. Figure (b) shows results for circular polarization's: left-hand (continuos line) and right-hand (dotted line). $\alpha=4$ and the values of the other parameters are the same as in figure Fig. [1] 


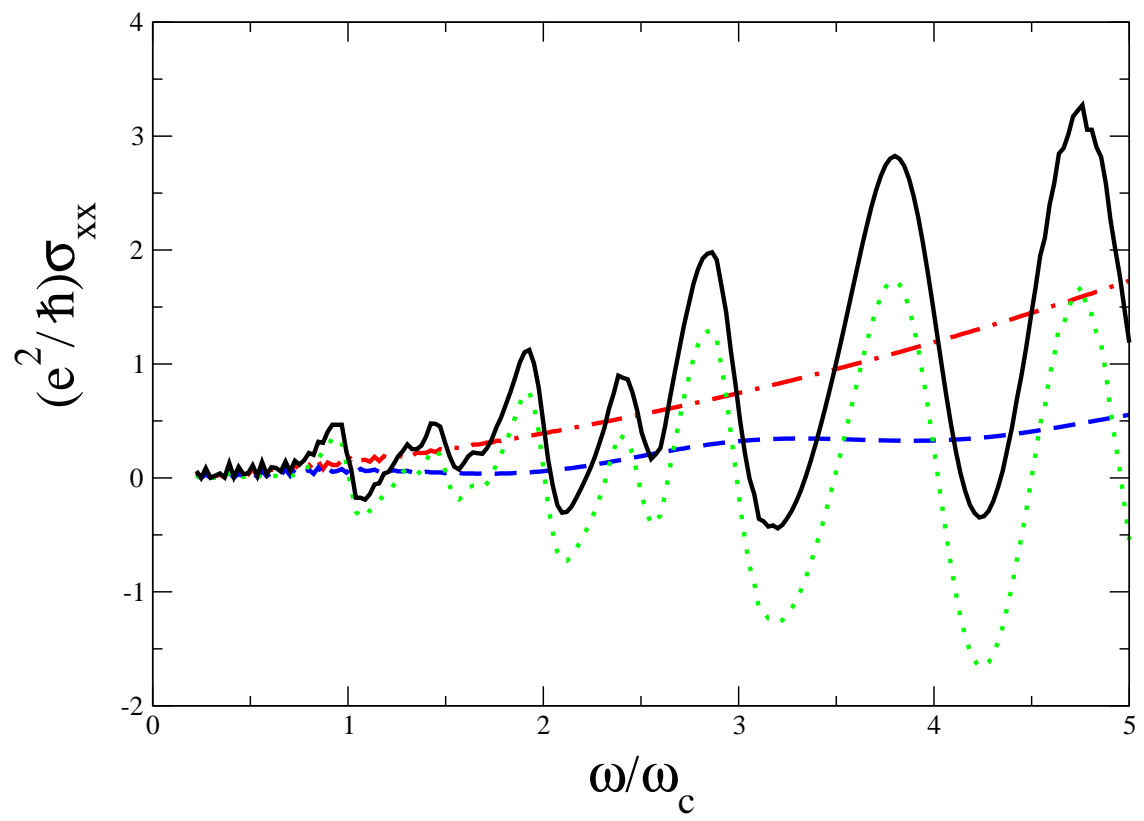

Figure 6. Contributions to the total $\sigma_{x x}$ (continuos line) vs. $\epsilon=\omega / \omega_{c}$ : dark contribution $\sigma_{x x}^{D}$ (dashed-dotted line) from Eq. (38), periodic modulation contribution $\sigma_{x x}^{M}$ (dashed line) obtained from Eq. (35) when the microwave field is switched-off, microwave-modulation contribution obtained as $\sigma_{x x}^{M M}-\sigma_{x x}^{M}$. The values of the other parameters are the same as in figure Fig. [1]

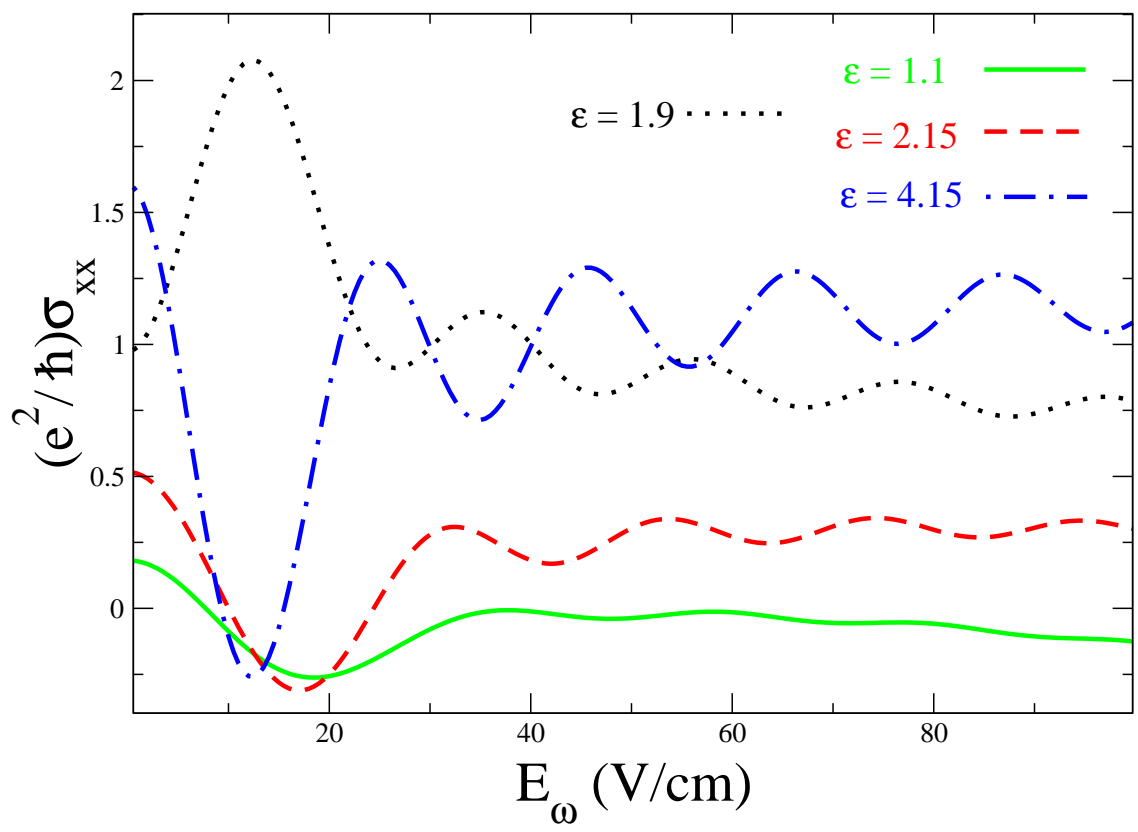

Figure 7. Longitudinal conductivity as a function of the microwave ac-electric field for various values of $\epsilon=\omega / \omega_{c}: \epsilon=1.1$ continuos line, $\epsilon=1.9$ dotted line, $\epsilon=2.15$ dashed line, and $\epsilon=4.15$ dashed-dotted line. The other parameters have the same values as in Fig. 1 
Photoconductivity in AC-driven modulated two dimensional electron gas in a perpendicular magnetic field 20

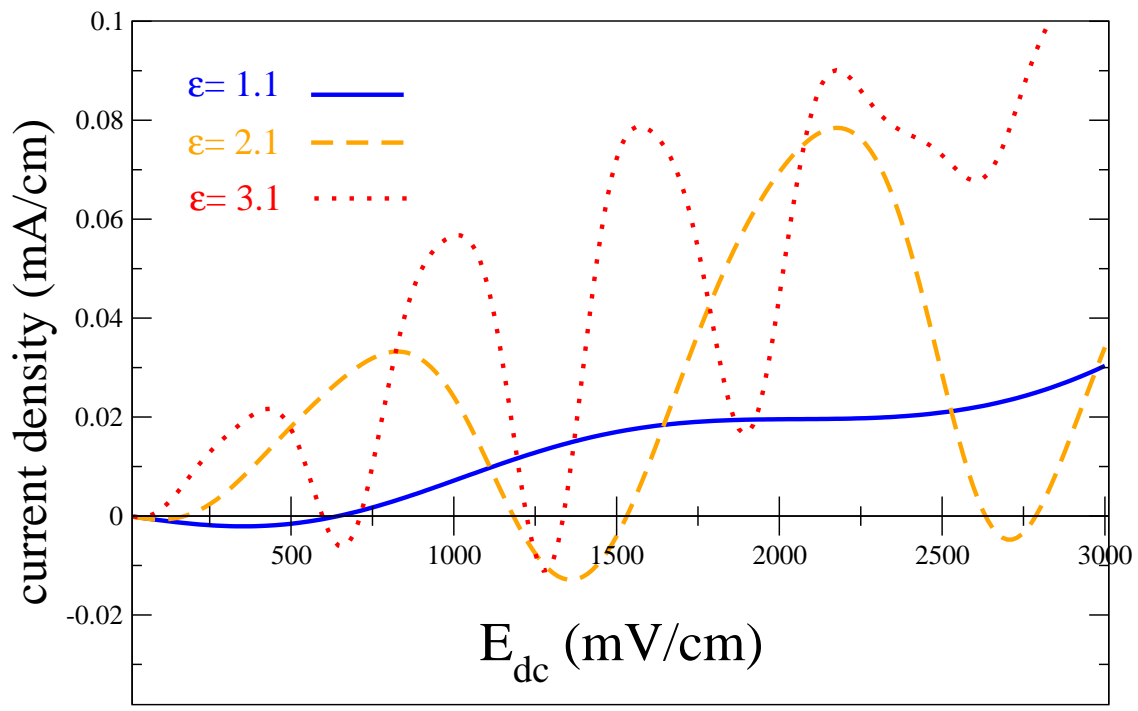

Figure 8. Current-voltage characteristics for the irradiated sample for various values of $\epsilon=\omega / \omega_{c}: \epsilon=1.1$ continuos line, $\epsilon=2.1$ dashed line, and $\epsilon=3.15$ dotted line. The microwave power is given by $\alpha=0.4$, the other parameters have the same values as in Fig. [1] 